\title{
Automated Image Mosaicing SySTEM WiTH ANALYSIS OVER VARIOUS IMAGE NOISE
}

\author{
Smriti Ayushi, Tejashwi Kalp Taru and Rajiv Kumar \\ Department of Computer Science and Engineering, Sharda University, \\ Greater Noida, Uttar Pradesh, India
}

\begin{abstract}
Mosaicing is blending together of several arbitrarily shaped images to form one large balanced image such that boundaries between the original images are not seen. Image mosaicing creates a large field of view using of scene and the result image can be used for texture mapping of a $3 D$ environment too. Blended image has become a wide necessity in images captured from real time sensor devices, bio-medical equipment, satellite images from space, aerospace, security systems, brain mapping, genetics etc. Idea behind this work is to automate the Image Mosaicing System so that blending may be fast, easy and efficient even if large number of images are considered. This work also provides an analysis of blending over images containing different kinds of distortion and noise which further enhances the quality of the system and make the system more reliable and robust.
\end{abstract}

\section{KEYWORDS}

RANSAC, SNR, Blending, Feature Extraction, Entropy

\section{INTRODUCTION}

Image mosaicing is the process of combining multiple overlapping images of same scene into a larger image. A mosaiced image is the composition of sequence of images, it can be obtained by finding out the coordinate relationship between the overlapping images. [1] Coordinate relationship refers to the relationship between the pixels of one part of image with another part of image which later on represents entire scene. The relationship depends on several parameters like matching corners and matching pixel values. Hence to form an entire large mosaic image of a scene, the pixel relationship must be transformed in such a way that can fit with other coordinates of other part of the scene. This process is known as homography estimation. [2] Automated image mosaicing system uses various procedures such as feature extraction, homography estimation and blending. Feature extraction includes the process to find out the corners in the image, then these corners must be translated in such a way that can fit with coordinates of another image they can be transformed, compared and analyzed in a common reference frame. Finally using this coordinate relationship the images are blended and final mosaiced image is obtained.

Mosaicing is the process of stitching several images together, hence the border and the matching features of image must be handled properly in such a way that border between the images are hidden and blended correctly. [3] There are lots of techniques which are used for the same purpose such as "Projected Mask". It is used to overcome the issues with cumulated errors between the 
borders of images. In this technique coordinate transformation is used between successive pairs of image patches. Hence this technique is highly resource and computation intensive. The basic challenge behind stitching images is to detect the common feature between two successive images like corners, edges and objects. After detection of such feature stitching is done by projecting one image onto another. This technique is useful for a wide range of scope like [4] microscopic image stitching to thermal image stitching but the core idea remains the same i.e. figuring out the features in image and then projecting one image onto another image. One of the novel idea that is also introduced in this process is recovering an image from noise, there are some techniques used to remove noises like smoothing the image using filters, but the main challenge remains same that there is a wide range of noise and system cannot remove every noise by just using a single filter, also sometimes it is possible that in the process of noise removal, image itself loses its features. This case is commonly caused due to the use of multiple filters or wrong filter with wrong type of noise.Hence an automated system which blends images irrespective of the quality and quantity of noise content has always been a requirement in this field.

Image mosaicing [6-10] is a major research area from the starting era of digital image processing. Anna Eivazi and Alexandar Kolesnikov in year 2015 have worked on Variance-preserving mosaicing of multiple satellite images for forest parameter estimation: Radiometric normalization. They have proposed a variance-preserving mosaic (VPM) algorithm that considers all images at the same time, minimizes overall error of the normalization and aims to preserve average variance of input images. Their proposed algorithm allows avoiding iterative pair-wise normalization, results in visually uniform mosaics while maintaining also the original image variance. Another remarkable work in this area is of B.Rousso and S. Peleg who have worked over video mosaicing to increase the visual field of view by pasting together many video frames.

In year 2000, Shmuel Peleg, Benny Rousso presented a new methodology to allow image mosaicing in more general cases of camera motion. They performed mosaicing by projecting thin strips from the images onto manifolds which are adapted to the camera motion. Richa Singh, in 2007 provided A Mosaicing Scheme for Pose-Invariant Face Recognition. She described a face mosaicing scheme that generates a composite face image during enrolment based on the evidence provided by frontal and semiprofile face images of an individual. Some researchers have also worked on Real-time Image Mosaicing. Nikhil Ranjan and Braj Bihari Soni chose RANSAC (Random Sample Consensus Algorithm) as their major technique in achieving image mosaicing and presented their work in year 2015.

Our system has been designed to use only one filter i.e. median filter as an optional step. It works by replacing the current pixel with the cumulative sum of neighbouring pixels. It also preserves the edge and features of an image in some cases. Hence it is possible to remove few noises from the image and then we can detect features and blend images even in the presence of certain quantity of noise. 


\section{MeTHODOLOGY}

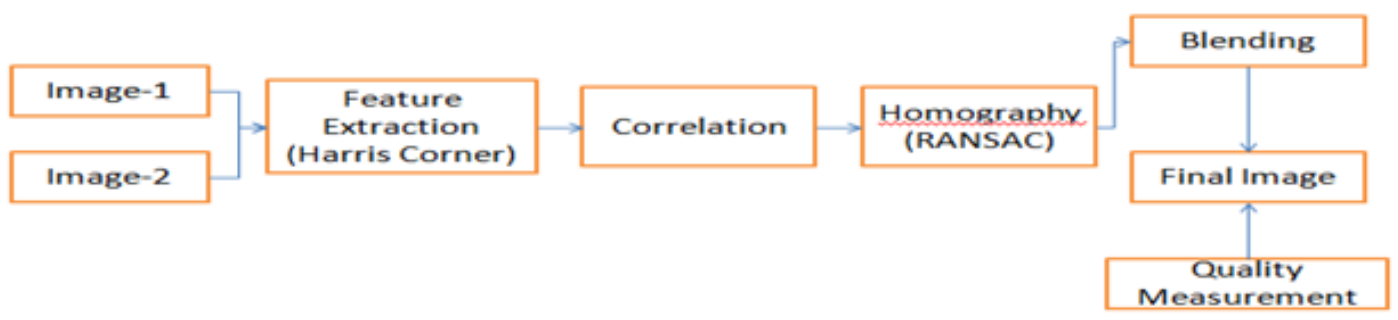

Figure1: Proposed model for image mosaicing system.

In the proposed model (as shown in Fig.1) the system will be using two images of a same scene to create a mosaic image. For this purpose, it will be using few algorithms including Harris Corner detection, Bidirectional cross correlation, Random Sample Consensus algorithm and alpha blending.

\subsection{Feature Extraction}

For feature extraction, the system needs to find matching points between different images of an environment. Due to the fact that if we know how two images relate to each other, we can use both images to extract information of them. Matching points refers to marking characteristics in the scene that can be recognized easily. [5] These are termed as characteristics features. Feature defines both corners and edges but here we are concerned of determining corners only. Corner detection is frequently used in motion detection, image registration, video tracking, image mosaicing, panorama stitching, 3D modelling and object recognition. Harris Corner Algorithm is used to detect corners in the input images. A corner can be defined as the intersection of two edges.
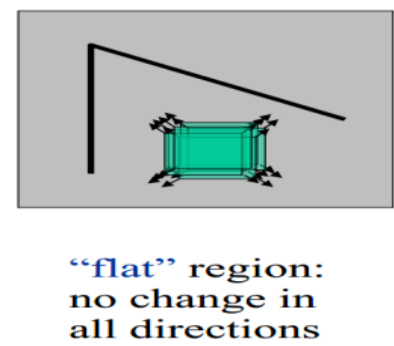

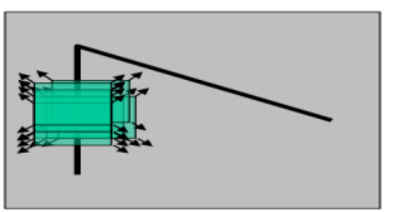

$$
\text { "edge": }
$$

no change along the edge direction

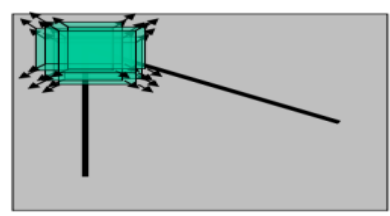

$$
\begin{aligned}
& \text { "corner": } \\
& \text { significant change } \\
& \text { in all directions }
\end{aligned}
$$

Figure2: Flat region, edge and corner defined

Harris corner detector gives a mathematical approach to determine which of the above case (Fig 2) holds. Consider a grayscale image I. We are going to sweep a window w(x, y) with displacement $\mathrm{u}$ in the $\mathrm{x}$ direction and $\mathrm{v}$ in the right direction and will calculate the variation of intensity:

$E(u, v)=\sum_{x, y} w(x, y)[I(x+u, y+v)-I(x, y)]^{2}$

(Equation 1) 
Where $w(x, y)$ is the window at position $(x, y), I(x, y)$ is the intensity at $(x, y)$ and $I(x+u, y+v)$ is the intensity at the moved window $(x+u, y+v)$. Shifting the window in any direction will yield a large change in appearance. Since we are looking for windows with corners, we are looking for windows with a large variation in intensity. Hence, we have to maximize the equation above, specifically the term:

$\sum_{x, y}[I(x+u, y+v)-I(x, y)]^{2}$

(Equation 2)

\subsection{Bidirectional Cross Correlation}

Correlation Algorithm is used to relate the corners found in the input images after Harris corner detection. Maximum bidirectional cross correlation rule has been used to determine matches between two images. The cross-correlation works by analyzing a window of pixels around every point in the first image and correlating them with a window of pixels around every other point in the second image. Points which have maximum bidirectional correlation will be taken as corresponding pairs. By using bidirectional cross correlation the designed system minimized the chances of having fake correlated points. When one point from the first image gives a positive correlation with another point of the second image, the system cross correlates that point with perspective of image two to image one. Then system selects those points which scored maximum cross correlation values.

Formulae for correlation ' $r$ ' can be expressed as:

$\mathrm{r}=(1 /(\mathrm{n}-1)) \cdot\left[\left(\sum_{\mathrm{x}} \sum_{\mathrm{y}}\left(\mathrm{x}-\mathrm{x}^{\prime}\right)\left(\mathrm{y}-\mathrm{y}^{\prime}\right)\right) /\left(\mathrm{S}_{\mathrm{x}}, \mathrm{S}_{\mathrm{y}}\right)\right]$

(Equation 3)

Where $\mathrm{n}$ is the number of pairs of data. $\mathrm{x}^{\prime}$ and $\mathrm{y}^{\prime}$ are the means of all the $\mathrm{x}$-values and $\mathrm{y}$-values and $S_{x}$ and $S_{y}$ are the standard deviation of all $x$ and $y$ values. To find the standard deviation, following equation can be used:

$\mathrm{S}_{\mathrm{x}}=\left(\left(\sum\left(\mathrm{x}-\mathrm{x}^{\prime}\right)^{2}\right) /(\mathrm{n}-1)\right)^{1 / 2}$

(Equation 4)

\subsection{Random Sample Consensus Algorithm}

Random sample consensus algorithm or RANSAC is a type of mathematical parameters model estimation method from a set of data which contains outliers, hence it is also known as outliers' detection method. [6] It is a non-deterministic algorithm because it produces a result only with certain probability and with this the probability is increased if more iteration on the data set is allowed. Data have inliers and outliers. Inliers are those data, whose distribution can be explained by some set of model parameters, but it may be subject to noise. Outliers are data that does not fit into any model, it may come from extreme values of noise. If fitting of a line is considered, assuming two dimensions to a set of observations which contains both inliers that is points and they have to be fitted in line. Outliers being points which cannot be fitted to this line. In this case RANSAC can produce a model which is computed from the inliers, provided that the probability of choosing only inliers in the selection of data is high. Due to probabilistic nature of RANSAC there is no guarantee for this situation but there are number of algorithm parameters which can be chosen to keep level of probability high. 


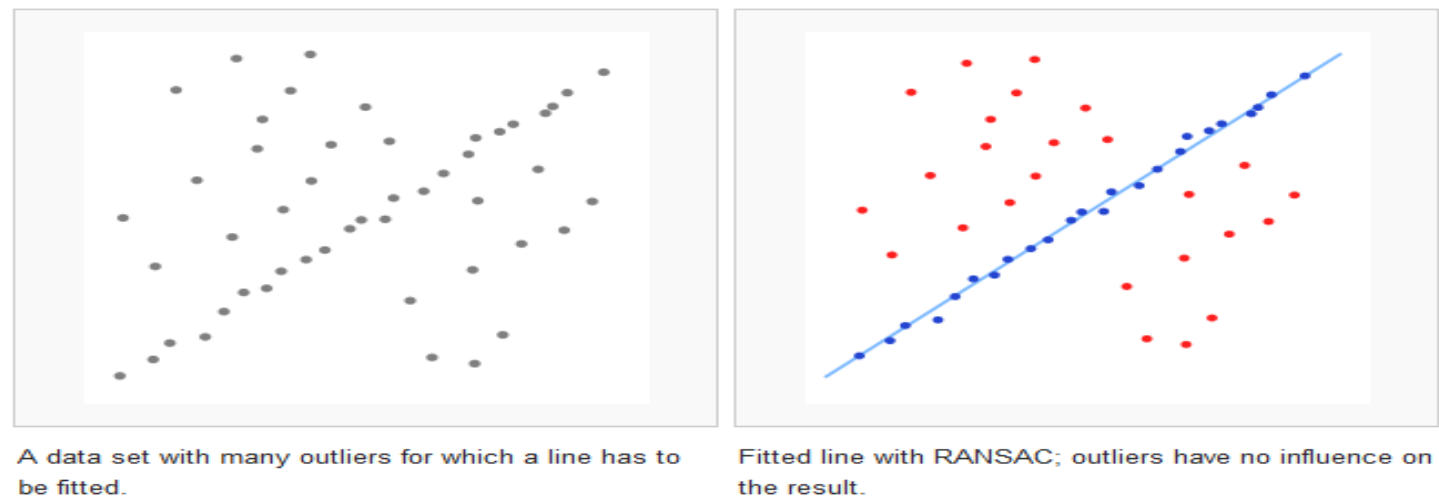

Figure3: RANSAC selected best fit Probabilistic Model

\subsection{Linear Gradient Alpha Blending}

It is the process of combining an image with a background to create the appearance of partial or full transparency. It is often useful to render image elements in separate passes, and then combine the resulting multiple 2D images into a single, final image paragraphs must be indented. [7] The alpha channel is a colour component that represents the degree of transparency (or opacity) of a colour (i.e., the red, green and blue channels). It is used to determine how a pixel is rendered when blended with another. The alpha channel controls the transparency or opacity of a colour. Its value are 0 for full transparency whereas 1 for full opacity. When a colour (source) is blended with another colour (background), e.g., when an image is overlaid onto another image, the alpha value of the source colour is used to determine the resulting colour. If the alpha value is opaque, the source colour overwrites the destination colour. If transparent, the source colour is invisible, allowing the background colour to show through. If the value is in between, the resulting colour has a varying degree of transparency/opacity, which creates a translucent effect. It is primarily used in Alpha Blending. Our system uses linear gradient alpha blending from the centre of one image to the other. The gradient blending works by simulating a gradual change in one image's alpha channel over the line which connects the centres of the two images.

\subsection{Algorithm}

For Image (X, Y)

Step 1: Extract feature using equation 1 and 2.

Step 2: Feature matching done using correlation equation 3 and 4.

Step 3: Homography estimation using RANSAC rule.

Step 4: Blending done using linear gradient alpha blending rule.

\section{EXPERIMENTS With NUMERICAL AND GRAPHICAL RESUlTS}

Dataset used for the implementation of algorithm and output of the algorithm is presented in Table 1. Also the histogram for the data analysis of the input and output are presented in Table 2 . 


\subsection{Observations and Results}

Mosaicing System is successfully merging or combining two images which are having some overlapped regions. (Refer Image set 1, 9, 10, and 11). Mosaicing System is taking care of proper blending even if one of the images is distorted. (Refer Image set 2). System is taking care of proper blending even is one of the image is subset of second image. (Refer Image set 2, 3, 6, 7, and 8). System is taking care of proper blending even when intensity variation in closely associated pixels is very high (Refer Image set 6). Example in this image, considering even a small image matrix, intensity value of each pixel is highly different from other pixels in same image matrix. System is taking care of proper blending even when second image is highly zoomed-In version of first image. (Refer Image set 7). System is taking care of proper blending even when one of the images is highly blurred subset of other image. (Refer Image set 8). Mosaicing System is taking care of proper blending even when one of the images is highly blurred subset of other image. (Refer Image set 11).

Signal to Noise ratio of the image input and output is tabulated in Table 3. [11] It can be observed from the analysis that the output image has approximately the same SNR value as of the two input images or if there is any decline in SNR value, it is up to a very smaller extent. Our mosaicing tool does not impact the noise quality of images to a noticeable extent. The quality of the input image that is provided to the system, the output image is given accordingly.

Entropy is the value to figure out the uncertainty i.e. nature of the image. Entropy value of input and output image is tabulated in Table 4. Low entropy image will have very little contrast and large number of pixels with similar pixel values, while high entropy images shows that image contains high contrast and lots of difference in pixel values.

Blur metric is a measure to find out the level of blur in image. Blur metric measure is tabulated in Table 5. This analysis is used to find out if the mosaicing process, especially the alpha blending which is changing the alpha values of images is making any changes to the blur level of output image or not.

Table 1. Output of Algorithm over Data Set

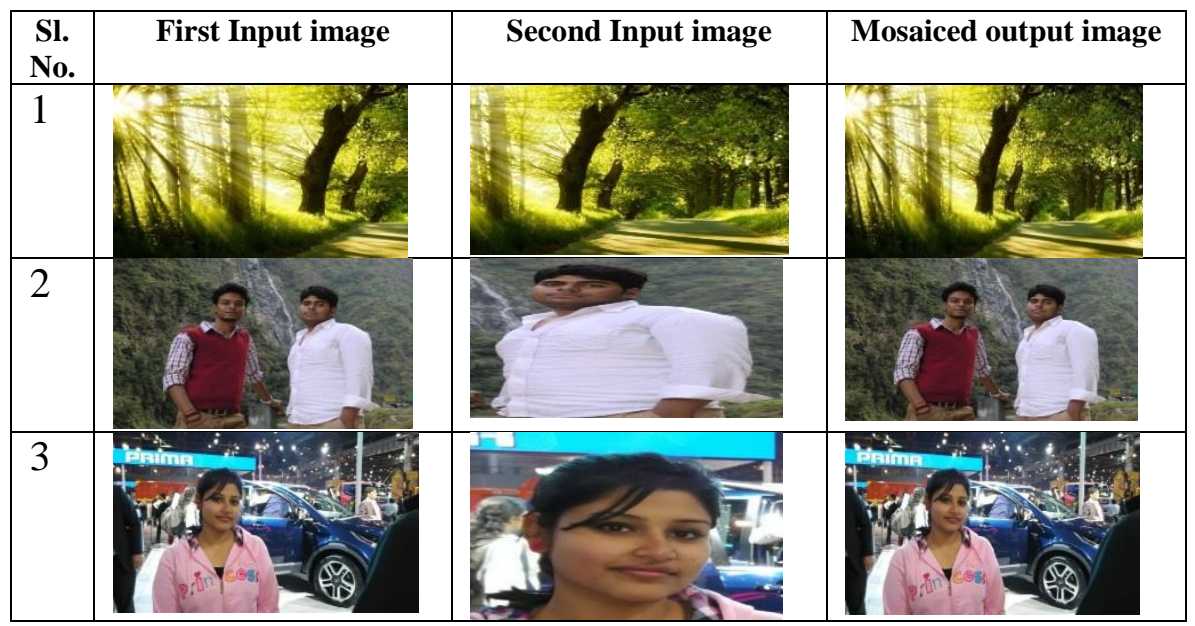


International Journal on Computational Science \& Applications (IJCSA) Vol.6, No. 3, June 2016

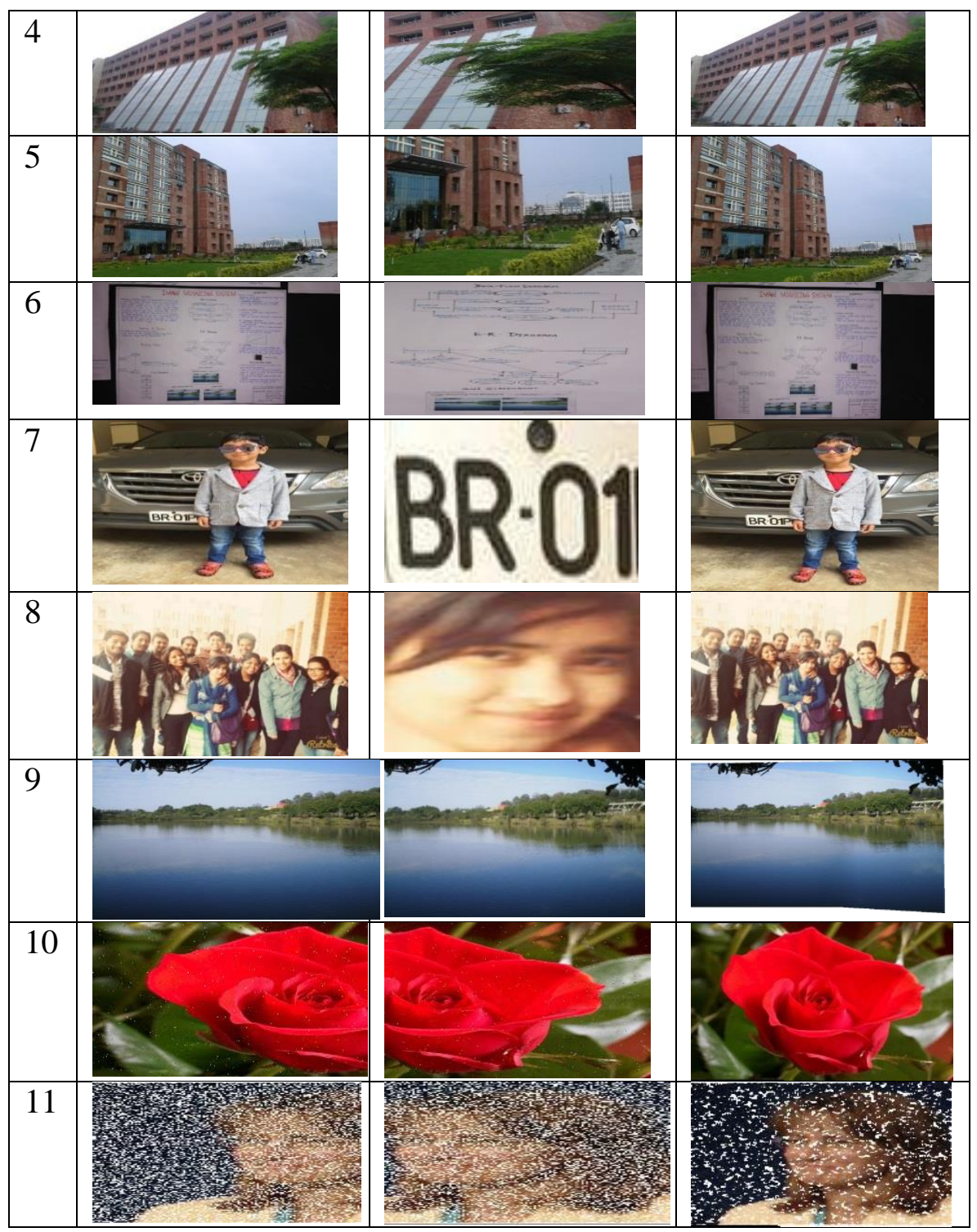

Table 2. Histogram of Input Data Set and Mosaiced Output

\begin{tabular}{|l|c|c|c|}
\hline $\begin{array}{c}\text { Sl. } \\
\text { No. }\end{array}$ & Input image 1 & Input image 2 & Mosaiced image \\
\hline 1 & & & \\
& & & \\
& & & \\
\hline
\end{tabular}


International Journal on Computational Science \& Applications (IJCSA) Vol.6, No. 3, June 2016

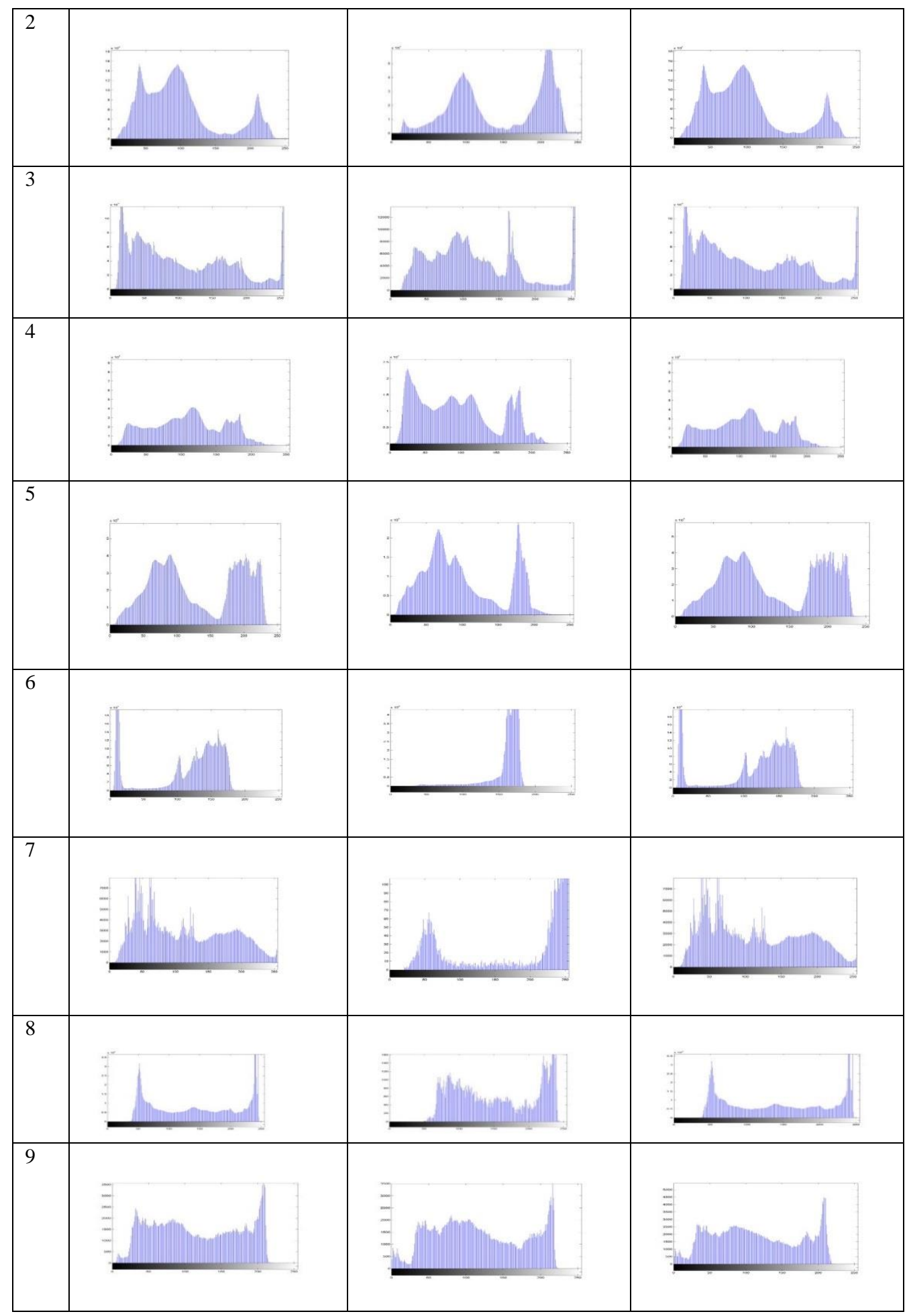


International Journal on Computational Science \& Applications (IJCSA) Vol.6, No. 3, June 2016

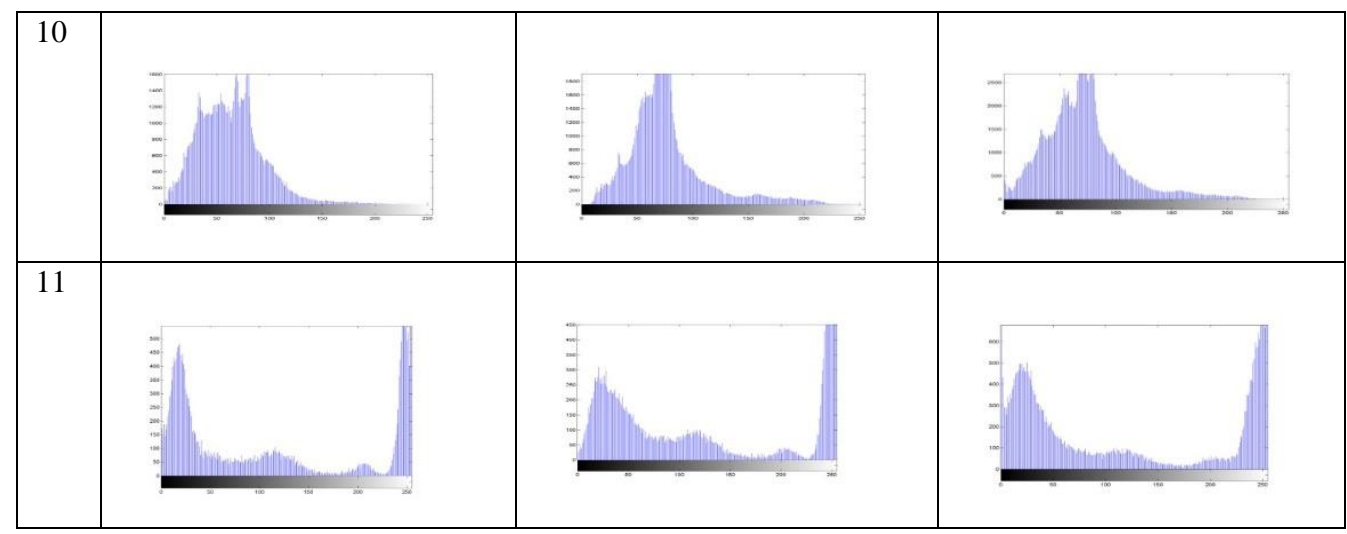

Table 3. Signal To Noise Ratio Analysis of Data

\begin{tabular}{|l|l|l|l|}
\hline S. No & SNR of Image One & SNR of Image Two & SNR of output image \\
\hline 1 & 12.8108 & 11.6365 & 12.8300 \\
\hline 2 & 10.7148 & 10.7846 & 10.7207 \\
\hline 3 & 11.6088 & 12.7368 & 11.6068 \\
\hline 4 & 11.2705 & 12.6914 & 11.2692 \\
\hline 5 & 10.3590 & 17.8004 & 10.7730 \\
\hline 6 & 11.7764 & 9.6033 & 11.7975 \\
\hline 7 & 10.6909 & 10.8503 & 10.6918 \\
\hline 8 & 12.0465 & 12.0140 & 11.6369 \\
\hline 9 & 10.4062 & 10.0790 & 10.5424 \\
\hline 10 & 8.1255 & 8.5420 & 9.8013 \\
\hline 11 & 10.2548 & 11.9724 & 11.1100 \\
\hline
\end{tabular}

Table 4. Entropy Analysis of Data

\begin{tabular}{|l|l|l|l|}
\hline S. No & Entropy of Image One & Entropy of Image Two & Entropy of output image \\
\hline 1 & 7.3991 & 7.2723 & 7.3971 \\
\hline 2 & 7.6945 & 7.5342 & 7.6961 \\
\hline 3 & 7.2594 & 7.4733 & 7.2609 \\
\hline 4 & 7.5594 & 7.3433 & 7.5591 \\
\hline 5 & 6.5805 & 5.3791 & 6.5497 \\
\hline 6 & 7.7342 & 6.8419 & 7.7305 \\
\hline 7 & 7.1153 & 7.3418 & 7.1267 \\
\hline 8 & 7.5890 & 7.6470 & 7.5521 \\
\hline 9 & 6.8608 & 6.8492 & 6.9584 \\
\hline 10 & 6.9543 & 7.1694 & 6.9584 \\
\hline 11 & 7.1278 & 6.5489 & 7.1551 \\
\hline
\end{tabular}

Table 5. Blur Metric Analysis of Data

\begin{tabular}{|l|l|l|l|}
\hline $\begin{array}{l}\text { S. } \\
\text { No }\end{array}$ & Blur Metric of Image One & Blue Metric of Image Two & $\begin{array}{l}\text { Blur Metric of output } \\
\text { image }\end{array}$ \\
\hline 1 & 11.3451 & 11.4349 & 10.2372 \\
\hline 2 & 15.1143 & 15.4973 & 14.8458 \\
\hline 3 & 12.0988 & 11.3454 & 10.8457 \\
\hline 4 & 10.9457 & 11.0396 & 10.3945 \\
\hline 5 & 13.1377 & 13.1102 & 12.8236 \\
\hline
\end{tabular}


International Journal on Computational Science \& Applications (IJCSA) Vol.6, No. 3, June 2016

\begin{tabular}{|l|l|l|l|}
\hline 6 & 9.4343 & 10.2329 & 9.1212 \\
\hline 7 & 12.4397 & 11.9326 & 11.2737 \\
\hline 8 & 11.2323 & 11.9993 & 11.1110 \\
\hline 9 & 10.4931 & 11.0033 & 10.0901 \\
\hline 10 & 14.2483 & 13.9878 & 13.1974 \\
\hline 11 & 11.1216 & 11.9363 & 10.5785 \\
\hline
\end{tabular}
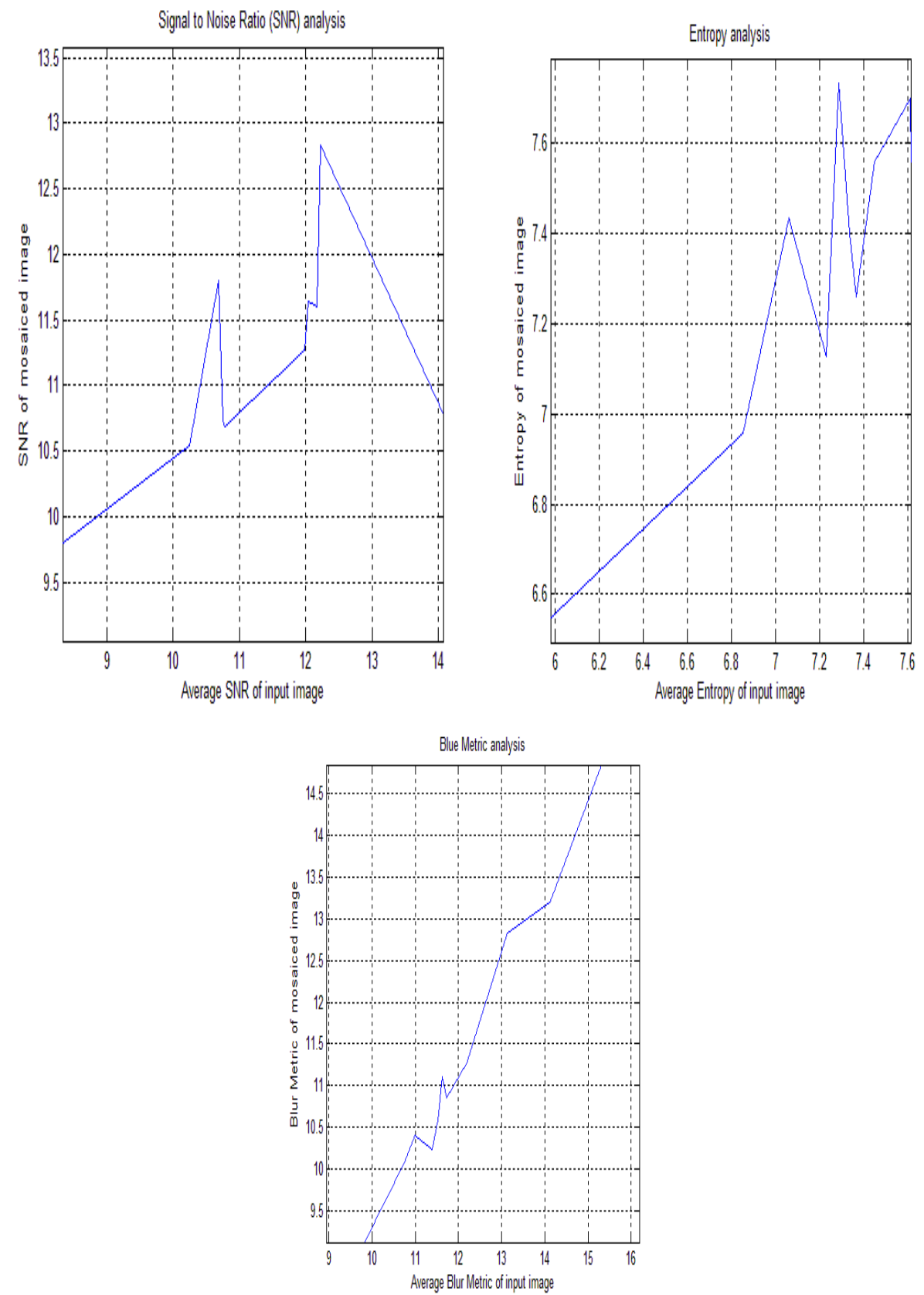

Figure 4: Graphical analysis of snr data, entropy data and blur metric data as tabulated above. 
International Journal on Computational Science \& Applications (IJCSA) Vol.6, No. 3, June 2016

\section{CONCLuSions}

All results clearly demonstrate that our system provides efficient mosaicing irrespective of various types of distortion present in image. Three image quality assessment techniques, namely SNR, Entropy and Blur Metric have been used. All of the result shows one final and concrete point that the quality of output image depends on the quality of input image supplied to the system. Hence all the algorithms that is used in this project does not effect in a high ratio the quality of result. Value of SNR is controlled in the output image if the value of SNR in input image is high, but this feature does not mean that the system will control a very high amount of SNR in the output image. Image Histogram comparison is done which stated that contrast is almost preserved as the input images and output image have nearly same histogram patterns. Similarly with the entropy and blur metric case their values come up to be approximately same or little declined when input and output are compared statistically and graphically. Hence perfect blending is achieved with single seamless mosaiced output for any kind of arbitrarily shaped input images (which can be taken from different angle too). A future work can be performed under this which can compare the results of the already existing mosaicing systems with the mosaicing tool built under this project. Quality assessment can be done from the mosaiced output of each system and their accuracy can be compared and significantly judged. Further the time and space complexity of the techniques and algorithms that are used in the project at each step for the processing of image can be found out and that time and space complexity can be tried to reduce as well. This Image Mosaicing that has been discussed can also be applied on 3D images in future.

\section{REFERENCES}

[1] Anna Eivazi And Alexander Kolesnikov, "Variance-Preserving Mosaicing Of Multiple Satellite Images For Forest Parameter Estimation: Radiometric Normalization", ISPRS Journal Of Photogrammetry And Remote Sensing, ELSEVIER, 2015.

[2] Dr. Ramesh B Inampudi, "Image Mosaicing", IEEE, 1998, 0-7803-4403-0/98.

[3] Shunichi Takeuchi, "Image Mosaicing Without Distortion Using Projected Mask For Image Digitization", IEEE, 0-7803-5467-2/99, 1999.

[4] Amar K. Bendale And Kantilal P. Rane, "M-Mosaicing And Thermo-Mosaicing Using Image InPainting", International Journal Of Computer Applications (0975 - 8887) Volume 95- No.21, June 2014.

[5] Darya Frolova, Denis Simakov, "Harris Corner Detector: Matching With Invariant Features",The Weizmann Institute Of Science, March 2004.

[6] Tsiombikas John, "An Efficient Technique For Image Mosaicing Using Random Sample Consensus Algorithm", International Journal Of Computer Applications (0975 - 8887) Volume 118 - No.16, May 2015.

[7] Shmuel Peleg, Benny Rousso, Alex "Mosaicing On Adaptive Manifolds", IEEE Transactions On Pattern Analysis And Machine Intelligence, Vol. 22, No. 10, October 2000.

[8] Richa Singh, Student Member, IEEE, Mayank Vatsa, Student Member, IEEE, Arun Ross, Member, IEEE, And Afzel Noore, Member, IEEE, "A Mosaicing Scheme For Pose-Invariant Face Recognition". IEEE TRANSACTIONS ON SYSTEMS, MAN, AND CYBERNETICS-PART B: CYBERNETICS, VOL. 37, And NO. 5, OCTOBER 2007. 
International Journal on Computational Science \& Applications (IJCSA) Vol.6, No. 3, June 2016

[9] Marc Vivet, Brais Martinez, Student Member, IEEE, And Xavier Binefa, "DLIG: Direct Local Indirect Global Alignment For Video Mosaicing", IEEE TRANSACTIONS ON CIRCUITS AND SYSTEMS FOR VIDEO TECHNOLOGY, VOL. 21, NO. 12, DECEMBER 2011.

[10] B. Russo, S. Peleg, "Universal Mosaicing Using Pipe Projection", Institute Of Computer Science, The Hebrew University Of Jerusalem 91904 Jerusalem, ISRAEL.

[11] Rajiv Kumar, Amresh Kumar, Pervez Ahmed, "A Benchmark Dataset For Devnagari Document Recognition Research", 6th International Conference On Visualization, Imaging And Simulation (VIS'13) Pages 258-63 